\title{
EVOLUTION OF INDOOR BULLET TRAP DESIGN
}

\author{
Filip Tikal \\ University of West Bohemia, Faculty of Mechanical Engineering, Regional Technological Institute, \\ Univerzitni 8, 30614 Pilsen, Czech Republic
}

\begin{abstract}
Taking into consideration the lack of standards for dynamic bullet and bullet fragments trap with difficult-defined energy, the issue is now solved by individual proposals of these devices. Its security is certainly the priority requirement of the construction. The device commonly ignores the investment cost and also operating cost due to lack of information on sizes of dynamic operational load.

In a systematic approach which is leading to the elimination of these negative aspects, it is necessary to perform a wide range of numerical simulation and experiments. They lead to the optimized dynamic bullet trap design proposal, which will be, based on experience obtained, possible to modify for required installation dimensions in particular application in practice. Finally, the concept is designed for environmental friendly approach to waste management.
\end{abstract}

Keyword: bullet trap; design; ballistic protection; explicit FEM analysis; experiments
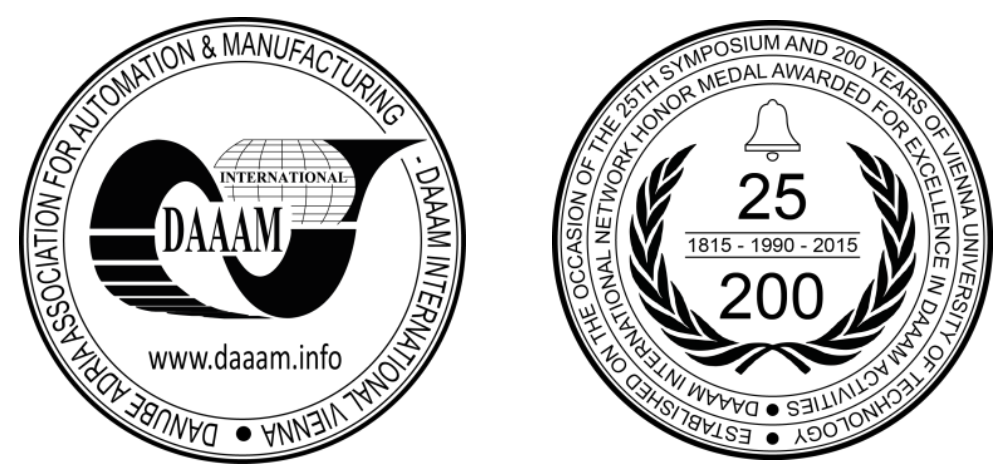

This Publication has to be referred as: Tikal, F[ilip] (2016). Evolution of Indoor Bullet Trap Design, Proceedings of the 26th DAAAM International Symposium, pp.0385-0390, B. Katalinic (Ed.), Published by DAAAM International, ISBN 978-3-902734-07-5, ISSN 1726-9679, Vienna, Austria

DOI:10.2507/26th.daaam.proceedings.052 


\section{Introduction}

When analyzing the current state of dynamic bullet trap constructions, an absence of standards and design recommendations for this issue was identified.

In practice, it is common to use only rough design knowledge without any verification by an analytical calculation, let alone numerical simulation and also the lack of knowledge of characteristics of the materials used. Safety and durability are solved only by oversizing constructions and thickness of materials is estimated. Furthermore, it does not take into consideration optimum joints. The material is often welded without regard to the lifetime of such a joint under dynamic impact stress.

They are also commonly applied in combination with non-metallic materials such as rubber, usually in the form of discarded tires, respectively conveyor belts etc. The above mentioned factors lead to high purchase price and expensive and environment-unfriendly bullet trap operation.

The motivation was therefore a systematic identification of a suitable construction along with both conventional and special materials and tests them for use in pre-selected defined load. The procedure was as in the field of numerical simulations and practical testing.

The concept of optimized dynamic bullet trap was verified by using an explicit finite elements solver. The explicit analysis allows simulating of extreme nonlinear behaviour in extreme situations, which lies on the limits or outside the area of solvable typical FEM programmes.

For testing of selected materials was at first performed a set of dynamic tests on specimens to determine material input data for numerical simulation. Subsequently was on the simulation model performed a set of analyzes of impacts and included the effects of possible penetration. Based on the simulation results the type of material was evaluated depending on the spatial orientation of the sample sheet metal and its damage the degree of protection. To validate the concept of bullet trap the set of experiments was conducted with using the test stand under real operating conditions.

\section{Conditions statement}

When analyzing requirements of the bullet trap was at first created statistics according to the regional requirements. This statistics was the further basis. The most frequently used limit caliber bullets, resp. their energy for civil rifles, were identified. Subsequently were chosen materials [1] with regard to the future intention to use a sandwich design and was defined speed and energy of bullets for a numerical simulation of the shooting distance, which according to the producer information complied with the conditions of the experimental testing.

\section{Used boundary conditions}

\subsection{Cartriges}

SAKO .308 Win 141A Racehead with the bullet weighing 10.9 grams, bullet type Racehead HPBT. It is a full metal jacket bullet with the lead core. The bullet is excellent in very thin casing and in bottom shaped as "boat tail", which gives the bullet very good ballistic characteristics.

\subsection{Dimensions of the samples and tested materials}

The dimensions of the samples of materials - A4 paper size.

a) Hardox 500 with a thickness of $8 \mathrm{~mm}$

This material is an abrasion resistant plate with a hardness of about $500 \mathrm{HB}$, intended for applications where demands are imposed on abrasion resistance made by SSAB Oxelösund, Sweden. Hardox 500 is not intended for further heat treatment. It has obtained its mechanical properties by quenching. The properties are not suited for applications requiring hot working at temperatures above $250^{\circ} \mathrm{C}$ since the material may then loose it's good properties. Welding of this material is not recommended.

b) S355 (CSN 11523) with a thickness of $10 \mathrm{~mm}$ and $12 \mathrm{~mm}$

This material is a non-alloy steel for structural purposes. Welding of this material is recommended.

\subsection{Geometry of testing stand}

Rake angles of samples on the stand: $90^{\circ}$ and $45^{\circ}$ to the horizontal axis Shooting distance: 50 meters 


\section{Numerical simulation}

\subsection{Explicit FEM theory}

The beginning of the development of explicit solvers goes back to the sixties. At that time began the development particularly at universities. The HEMP programme, which had freely available code, was the basis of gradually developed these days used software. Explicit time integration is suitable for a simulation of processes with large deformations and reshaping. There is a better chance of capturing nonlinear behavior of material and fracture. The explicit solvers are generally better suited for tasks with complex contact situations. Thanks to these characteristics are the explicit solvers determined to solve conflict tasks, so called crashes, bullet holes etc.

Explicit code basically comes from Newton's second law of motion. This is the equation of motion incorporated in the matrix form (1). This equation is defined at the given time. In order to keep the balance of the dynamic forces, the relationships described below must be met [2].

$$
\left\{a_{t}\right\}=[M]^{-1}\left(\left\{F_{t}^{\text {ext }}\right\}-\left\{F_{t}^{\text {int }}\right\}\right)
$$

Here is $\left\{a \_t\right\}$ the acceleration vector (at time $\left.t\right),[M]$ is the weight matrix, $F \_t \wedge$ ext a vector of external forces acting on the body and $F_{-}{ }^{\wedge}{ }^{\wedge}$ int is the vector of internal forces.

After definition of the internal forces and adding some basic elements, it is possible to create an equation for the numerical solution in the following format (2). The element $\left\{\mathrm{F}^{\wedge}\right.$ houg $\}$ was added to prevent hourglassing and $\left\{\mathrm{F}^{\wedge} \mathrm{cnt}\right\}$ as vector of contact forces. Furthermore $\left\{\sigma_{-} n\right\}$ is internal tensions matrix, $[B]$ is matrix of reshaping elements.

$\left\{F_{t}^{\text {int }}\right\}=\sum\left(\int_{\Omega}[B]^{T}\left\{\sigma_{n}\right\} d \Omega+\left\{F^{\text {houg }}\right\}\right)+\left\{F^{\text {cnt }}\right\}$

The solvers based on the explicit code are conditionally stable. It means that they are stable only under certain conditions. This is mainly about the time step size. It is related to propagation of voltage waves in the material (see following equation (3). Here is $c$ the wave's propagation speed in the material, $l$ is the characteristic size of the element, $E$ is the module of the material elasticity and $\rho$ the density of the material.

$t_{\text {calc }} \leq t_{\text {krit }}=\frac{l}{c}=l \sqrt{\frac{\rho}{E}}$

The big advantage of the explicit method is the use of elements with a single integration point. The advantages of this method are, however, redeemed by reduced stability of the calculation. If the element is deformed symmetrically, there is no corresponding change in internal energy. In the calculation result are therefore the typical imbalance between kinetic and internal energy of the system. This numerical error is called hourglassing. It is obvious that during the dynamic calculations must be always controlled the total energy. As critical it is considered the increase in hourglassing energy over $5 \%$ of the total energy of the system. In extreme cases of hourglassing increase may even collapse calculation. To limit the hourglassing occurrence are used different methods.

\subsection{Explicit FEM solver Pam-Crash}

Pam-Crash is a FEM solver that is part of the software package VPS (Virtual Performance Solution) produced by ESI Group. [2] The software is used for crash simulation and safety assessment. This software is most commonly used in the automotive industry.

The software has been developed since 1978 and is connected with the early car crash simulations. The software is based on the finite element method (FEM) and allows modeling of complex geometry with a broad range of different types of finite elements. The programme offers a wide range of linear and nonlinear materials including viscoplastic, foam and multi-layered composites, including models of failure [4].

Thanks to the usage of the explicit formulation FEM, is the software suitable for simulation of nonlinear tasks with a large number of contacts (mainly based on the penalty algorithm).

This software was selected based on references from the field of defense, because it allows solving tasks such as the performance of munitions with respect to explosion, cratering and the simulation of kinetic energy penetrators.

\subsection{Description of numerical simulations}

Initially was completed a simulation model, subsequently were set boundary conditions and implemented material data from dynamic tests on specimens.

The following description of the simulation model is valid for one situation of boundary conditions. Other models were created as variants. These variants were modifications of this debugged pilot case. 
The bullet consisting of a lead core and a brass surface casing crashed into a metal sheet of material S355 with the thickness of $10 \mathrm{~mm}$. Initial bullet speed was $779 \mathrm{~m} / \mathrm{s}$ [5]. To create the model, we used linear quadratic elements with eight nodes. The average size of the element is about $0.5 \mathrm{~mm}$. Material model number 19 "elastic-plastic-withdamage-failure" was chosen as a representative.

All three materials were defined based on this model. Flexible behavior was defined on the basis of stressstrain curves. Various stress-strain curves are defined for various values of strainrate. Thanks to that, the strengthening of the material during rapid deformation was taken into account.

As a criterion, it was considered maximum plastic strain element for elimination. Critical time step is defined by characteristic element size, young modulus and stiffness $\Delta \mathrm{t}=8,9 \mathrm{e}^{-6} \mathrm{~ms}$. Time of simulated process duration is 0.14 ms.

\subsection{Calculation of material model using JMatPro}

JMatPro is a commercial software package which is based on CalPhaD (Calculation of Phase Diagrams) methodology and extended by various models which allow calculation of materials properties. It is now widely used in steel industry and also in materials property data generation for FEM simulations [6].

This software was used for calculations of various stress-strain material curves for defined various values of strainrate. The term flow-stress is frequently used for these curves in steel industry. Input data required for calculating flow stress include chemical composition of the material and information about one of its basic mechanical properties (yield strength, ultimate strength, hardness or grain size). Input data were measured on devices: ZEISS EVO25 with Oxford Instruments detector - chemical composition and WOLPERT Vickers tester 432-SVD - hardness. See Table Nr. 1. - Fe and harmful attend trace-elements are the rest up to $100 \%$ of the chemical composition.

The results computed by JMatPro can be considered as successful for general steel and in case of immediate need of material stress-strain material curves. See Figure Nr. 1. Material model prepared by the JMatPro can be refined using FEM software DEFORM which is suitable for simulations of technological processes. This comprehensive approach will bring the influence of the technological process history in material model, which can be used then for further simulations such as the simulation of impacts.

\begin{tabular}{llllll}
\hline Material & \multicolumn{3}{c}{ Chemical composition } & \multicolumn{2}{l}{ Hardness } \\
\hline & C \% & Cr \% & Mn \% & Si \% & \\
Hardox 500 & 0,25 & 0,60 & 0,70 & 0,30 & 52 HRC on surface, 42 HRC under surface \\
S355 & 0,10 & & 1,50 & & $157 \mathrm{HV}$ \\
\hline
\end{tabular}

Table 1. Results of chemical and hardness analysis
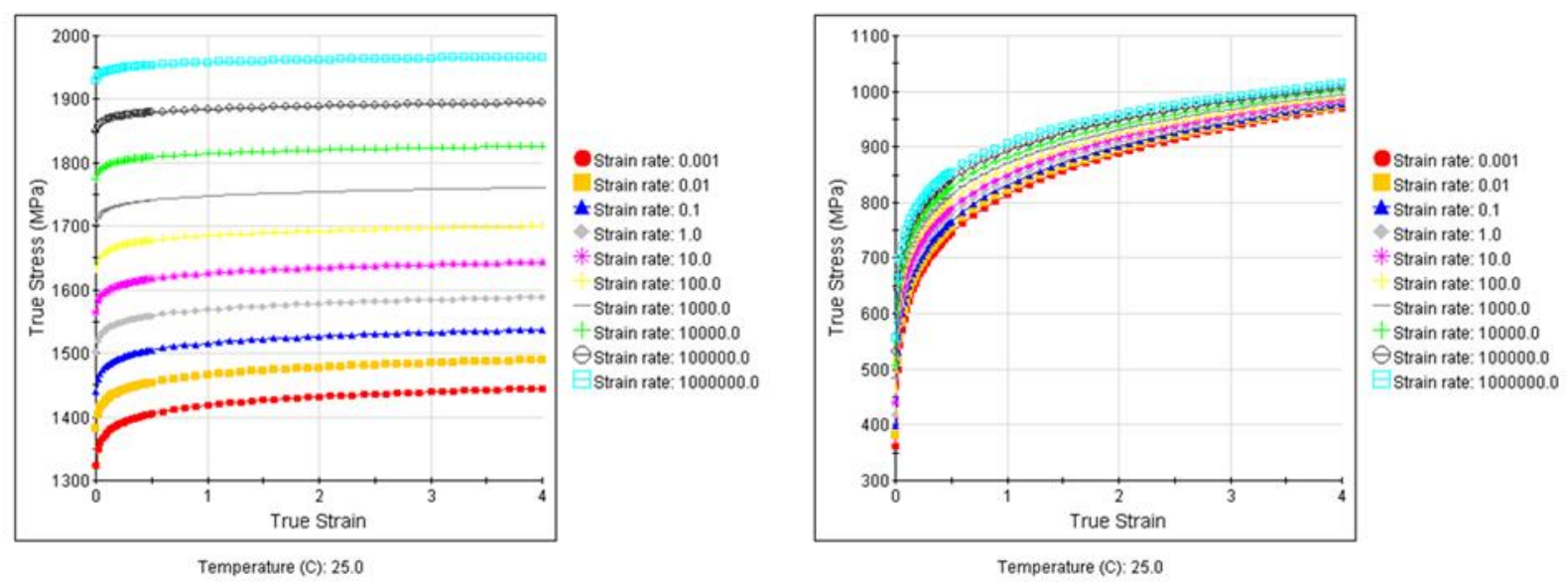

Fig. 1. Results of the material model simulations - Hardox 500 and S355

\subsection{Results of numerical simulations}

The results of simulation give a good knowledge of the resistance of the plate of steel material. It is very good visible a hole forming in the entrance of a bullet. For a board with the thickness of $10 \mathrm{~mm}$ at a right angle to the bullet trajectory there is the complete penetration of the projectile. The bullet speed is due to the loss of energy during penetration reduced by $70 \%$. 
When simulating bullet impact with a plate inclined by an angle of $45^{\circ}$, with respect to the bullet trajectory, does not come to penetration. This result corresponds to the result of the experiment. It is apparent that the board inclination significantly increases its resistance.
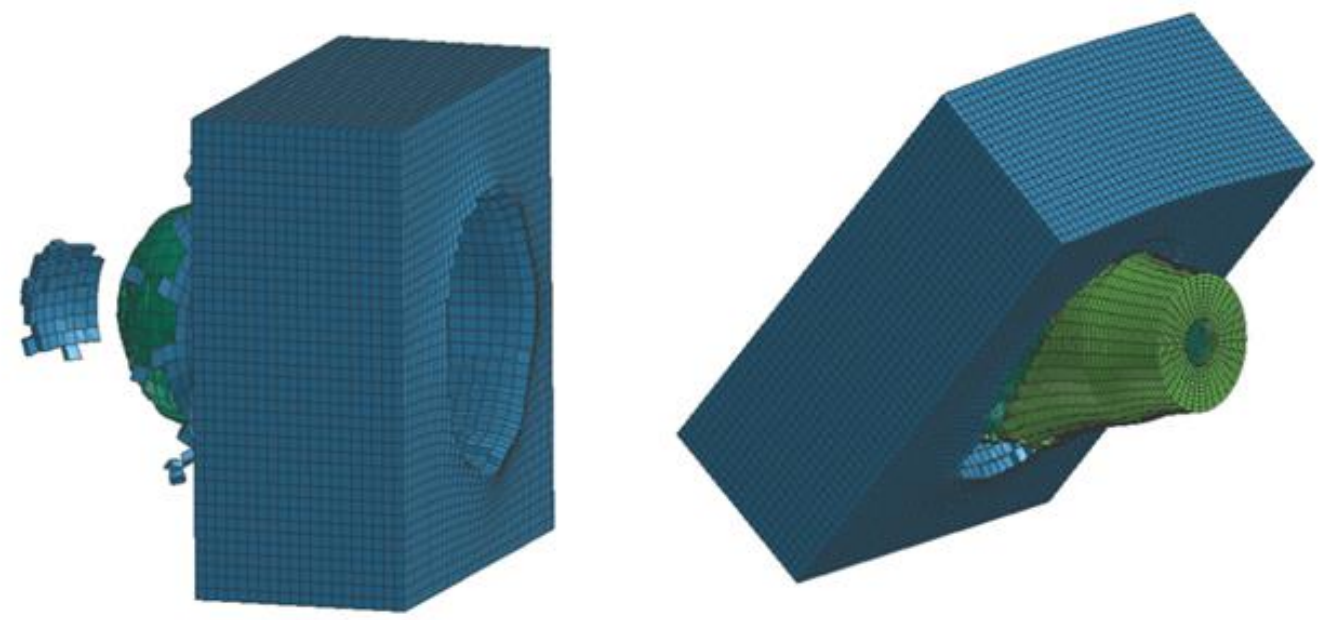

Fig. 2. Results of the simulations - the plate from material S355 with thickness $10 \mathrm{~mm}$ inclined by angle $90^{\circ}$ and by angle $45^{\circ}$

\section{Experiments}

For the validation of numerical simulations was performed set of experiments using the testing stand in real operating conditions. The shooting was conducted at a distance of $50 \mathrm{~m}$ with a sniper rifle of following parameters:

Remington 700 Police (short action) [7] Caliber .308 Win., Barrel Length 26" Standard Twist 12".

For the shooting experiments were used specially made rectangular metal sheets of paper size A4 positioned immovably in the special stand, which allows to select the angle of inclination of the material samples.

On the plate were shot two times for single variant of combination of metal sheet material and the angle of inclination. Each score was documented. See Figure Nr. 3.

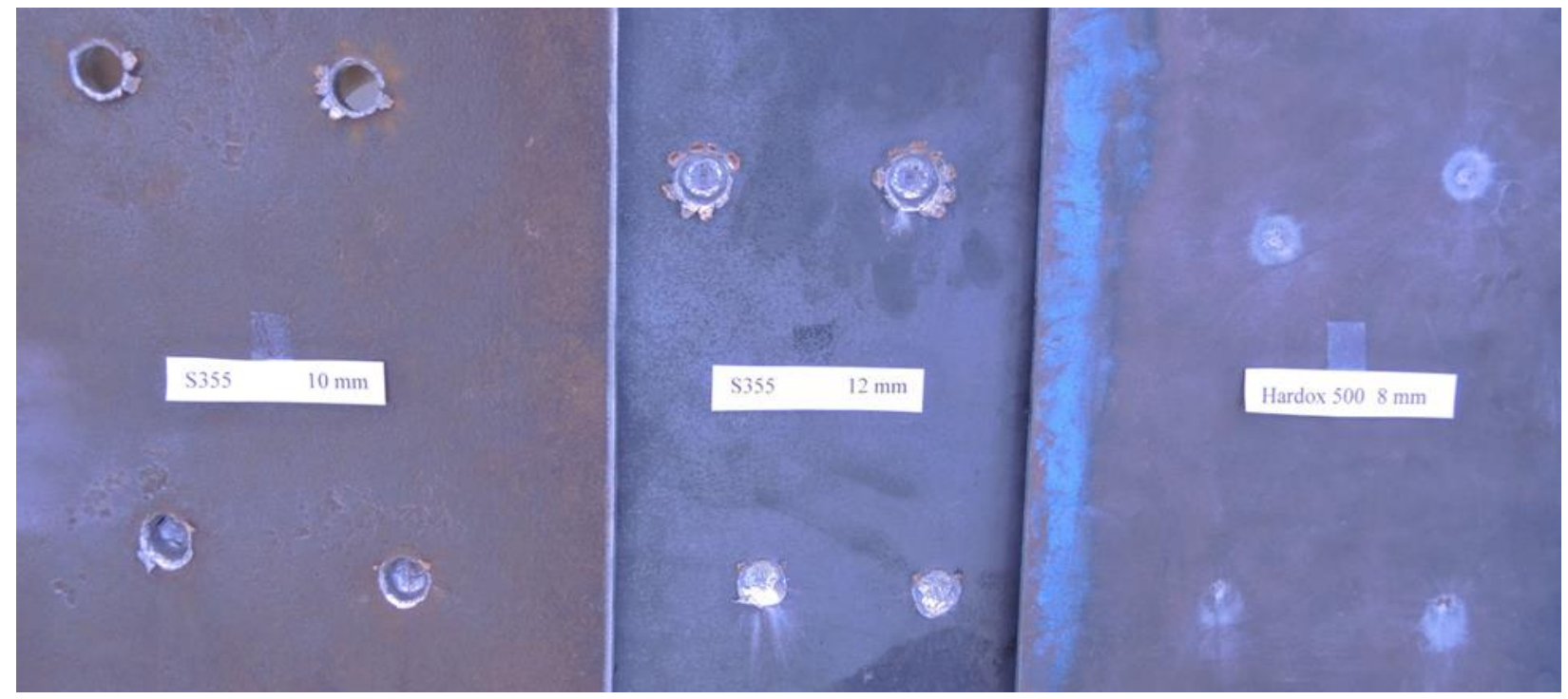

Fig. 3. Results of the shooting experiments - the plates inclined by angle $90^{\circ}$ in the top line of hits and by angle $45^{\circ}$ in the bottom line

During the shooting experiment were achieved results that confirmed the assumptions ballistic-resistant of metal sheet calculated by using numerical simulations. See Table Nr. 2. 


\begin{tabular}{|c|c|c|c|}
\hline Material & Angle & Thickness & Results for 2 shots \\
\hline S355 & $90^{\circ}$ & $10 \mathrm{~mm}$ & penetration \\
\hline S355 & $90^{\circ}$ & $12 \mathrm{~mm}$ & cup deformation \\
\hline Hardox 500 & $90^{\circ}$ & $8 \mathrm{~mm}$ & micro deformation \\
\hline $\mathrm{S} 355$ & $45^{\circ}$ & $10 \mathrm{~mm}$ & cup deformation \\
\hline S 355 & $45^{\circ}$ & $12 \mathrm{~mm}$ & cup deformation \\
\hline Hardox 500 & $45^{\circ}$ & $8 \mathrm{~mm}$ & abrasion only \\
\hline
\end{tabular}

Table 2. Results of experiments

\section{Conclusion}

Numerical simulations provided the assumptions that were subsequently verified by the shooting experiments. These findings will be used for a selection of materials and design of optimized dynamic bullet trap. Present simulations and experiments are in good results accordance in overview. This means results like penetration, deformation and abrasion but their geometry in results of simulations and experiments is different, therefore future refinement will be done [8].

For future research is planned to extend the analysis to other caliber, inclination angles and thickness [9] of material samples. The tests of other materials will be also included. The materials will be selected with regard to price. It also assumes the development and use of special rolled sandwich materials.

Further to the shooting conduct will be necessary to include an electronic gate to measure the speed of bullets (chronograph) and install to the testing stand a high speed imaging camera and a speed thermal imaging camera for more detailed evaluation of impacts dynamic process with simultaneous temperature monitoring.

The results achieved will be prepared for the evaluation software, which enables effective proposals of bullet trap mainly for civilian and military indoor shooting ranges and ballistic departments.

\section{Acknowledgements}

The present contribution has been prepared under project LO1502 'Development of the Regional Technological Institute' under the auspices of the National Sustainability Programme I of the Ministry of Education of the Czech Republic aimed to support research, experimental development and innovation.

\section{References}

[1] J. Hub, J. Komenda, Ballistic Resistance of Steel Plate Hardox upon Impact of Non-Penetrating Projectiles. in: Advances in Military Technology Vol. 4, No. 2, December 2009, pp. 79-91.

[2] T. Belytschko, J.L. Lin, C.S. Tsay, Explicit algorithms for the nonlinear dynamics of shells. in: Computer Methods in Applied Mechanics and Engineering 42 (1984), pp. 225-251.

[3] Impact and high velocity impact [WWW] http://virtualperformance.esi-group.com/applications-impact-and-highvelocity-impact-analysis (29.04.2015).

[4] E. Mestreau, R. Lohner, Airbag Simulation Using Fluid/Structure Coupling. 34th Aerospace Sciences Meeting \& Exhibit, Reno, NV, January 1996.

[5] Sako Ballistics [WWW] http://luoti.sako.fi/Ballistics/index.jsp (29.04.2015).

[6] U. Diekmann, Calculation of steel data using JMatPro. COMAT2012, 21. - 22. 11. 2012, Plzeň, Czech Republic.

[7] Remington 700 Police [WWW] http://www.remingtonle.com/rifles/700p.htm (29.04.2015).

[8] J. Buchar, J. Voldřich, Terminal ballistics (in Czech). Praha: Academia, 2003, ISBN 80-200-1222-2.

[9] J. Hub, J. Komenda, Determination of the Protective Barrier Thickness of the Restraint System. in: Advances in Military Technology Vol. 8, No. 2, December 2013, pp. 63-70. 